\title{
ÍNDICE PARA LA MEDICIÓN DE LA COMPETITIVIDAD EN COLOMBIA
}

\author{
Index for measuring of competitiveness in Colombia \\ Natalia María Acevedo Prins \\ Magíster en Ingeniería Industrial. Institución Universitaria ESUMER, Medellín - Colombia, \\ natalia.acevedo4@esumer.edu.co \\ Luis Miguel Jiménez Gómez \\ Especialista en Ingeniería Financiera. Universidad Nacional de Colombia, Medellín - Colombia, \\ lumjimenezgo@unal.edu.co
}

\begin{abstract}
Cómo citar/ How to cite
Acevedo, N. y Jiménez, L. (2015). Índice para la medición de la competitividad en Colombia. Revista CEA, 1(2), 109-121.
\end{abstract}

Recibido: 12 de mayo de 2015

Aceptado: 25 de junio de 2015

\section{Resumen}

Esta investigación presenta una metodología para la construcción de un índice compuesto para medir la competitividad como factor de crecimiento económico para Colombia, a partir de los modelos de competitividad de Michel Porter y el trabajo de Cho y Moon. Los índices actuales presentan falencias en la construcción, desde la parte teórica o desde el manejo estadístico. Es por esto que se implementó el análisis factorial, herramienta estadística que permite una construcción objetiva evitando la inserción de subjetividades en la ponderación y agregación de variables. También, la utilización de datos duros provee a la investigación de mayor confiabilidad. Por medio de esta construcción se evidenciaron los esfuerzos que realiza el país para mejorar la competitividad apoyada por la estabilidad macroeconómica, sin embargo rezagada por la seguridad y salud pertenecientes a la institucionalidad. Los resultados concuerdan con las recomendaciones de los estudios económicos que hace la Organización para la Cooperación y Desarrollo Económico para Colombia.

Palabras clave: Índice compuesto, competitividad, análisis factorial, diamante de la competitividad.

\begin{abstract}
This research presents a methodology for the construction of a composite index to measure competitiveness as a factor for economic growth in Colombia, from competitiveness models by Michel Porter and the work of Cho and Moon. Current rates have shortcomings in construction, from the theory or the statistical management. That is why a factor analysis was developed, a statistical tool that allows an objective construction, avoiding mistakes from including subjectivity in the weighting and aggregation of variables. Also, the use of hard data provides greater reliability to the research. Thanks to this construction the efforts of the country to improve competitiveness supported by macroeconomic stability were evident, nevertheless; lagging behind due to the safety and health belonging to the institutions. The results are consistent with the recommendations of the economic studies presented by the Organization for Economic Co-operation and Development Colombia.
\end{abstract}

Keywords: Composite index, competitiveness, factorial analysis, diamond of competitiveness. 


\section{INTRODUCCIÓN}

Entre los gobiernos y los líderes industriales existe un interés por encontrar las razones por las que las naciones $u$ organizaciones fracasan (Xia, Liang, Zhang, \& Wu, 2012), por esto, la competitividad se convierte en tema de debate político- económico en busca de la prosperidad de las naciones. (Delgado, Ketels, Porter, \& Stern, 2012).

Existen varios índices que son tomados como referencia por los gobiernos para el crecimiento económico y la competitividad de las naciones, siendo el de mayor aceptación a nivel mundial es el Índice de Competitividad Global, desarrollado por el Foro Económico Mundial, (WEF, por sus siglas en inglés) y reportado cada dos años, el segundo índice es el World Competitiveness Yearbook diseñado por el Instituto Internacional para el Desarrollo Gerencial, (Institute for Management Development, IMD), y por último se encuentra el Industrial Development Scoreboard desarrollado por Archibugi y Coco (Archibugi \& Coco, 2005).

Los índices expuestos tienen problemas en la construcción, desde la parte teórica o desde el manejo estadístico. La principal crítica encontrada en la literatura para estos índices es la falencia en el método de agregación y ponderación de variables debido a que son realizadas por el criterio de expertos, lo que brinda subjetividades a la medición, además, los índices mundiales son diseñados como primera instancia para los países desarrollados, y de esta forma, las características que no cumplen los países en vía de desarrollo, como ocurre con las economías latinoamericanas, son rezagadas con un valor cero afectando el rango donde se ubican estos países en el índice.

Esta investigación construye un índice de medición de la competitividad para el crecimiento económico de países como Colombia, con base en el modelo de Michael Porter y Cho y Moon, con el fin de obtener una medición objetiva para la competitividad del país, ya que las actuales son construidas con base en subjetividades de los creadores. Para evitar esto, se procede a la revisión del marco teórico que proveerá las variables a ingresar, se aplicará la metodología para construcción de indicadores sintéticos de la Organización para la Cooperación y Desarrollo Económico - OCDE, haciendo uso del análisis factorial como criterios multivariados y también como técnica para de agregación y ponderación.

\section{MARCO TEÓRICO}

La competitividad es un concepto que genera diversos debates. Aunque en términos empresariales, el concepto es claro, las inconformidades se generan cuando se habla de competitividad en el ámbito nacional; empero, la competitividad se analiza desde distintos ámbitos. Es sin embargo, la competitividad de las industrias la que presiona la competitividad en el entorno industrial a nivel nacional (Kao et al., 2008; Sanjaya Lall, 2001; Lira, 2005).

El concepto de la ventaja competitiva está basado en la productividad y en factores que la determinan. La productividad es la relación entre los resultados obtenidos de un sistema para producir bienes y servicios y los recursos usados para obtener este resultado, en otras palabras, el uso eficiente, o relación entre los resultados obtenidos y los recursos usados y el tiempo que toma obtenerlos (Krugman \& Obstfeld, 2000).

Weymouth y Feinberg (2011) indican que países competitivos son aquellos que luchan por políticas asociadas a una mayor productividad manteniendo estable la población. Argumentan que la productividad sube si el valor de bienes y servicios aumenta, o si los bienes y servicios se producen de manera más eficiente. Destacan que los factores determinantes para lograr esta competitividad son la inversión y la producción eficiente. 
M. Porter (1990) postula que las teorías económicas clásicas no son suficientes para entender la competitividad de las naciones asumiendo que el papel de las industrias y la estrategia empresarial son importantes para diferenciar un país de otro. En este caso, la teoría económica moderna surge como respuesta a un mundo globalizado, donde existe mayor concentración empresarial y mayor cantidad de empresas multinacionales que trabajan en diferentes países, las compañías no solo trabajan para satisfacer la demanda doméstica, sino que también compiten por espacios en mercados extranjeros contribuyen; por ello las teorías clásicas de crecimiento económico no expliquen bien el fenómeno (Ramos, 2001).

Con la idea de dar solución a las disyuntiva existente en temas de teorías económicas para tratar la competitividad, Michael Porter (Porter, 1990), crea el modelo más usado en términos de medición de la competitividad: diamante de la competitividad nacional, con el que se esperaba que diera respuesta al interrogante ¿Por qué ciertas empresas ubicadas en determinadas regiones o países, eran capaces de innovar y porqué otras no? Es decir, que este modelo busca encontrar cuáles son los factores o variables que hacen que las industrias crezcan y se desarrollen en menor tiempo que otras (Grant, 1991). La respuesta al interrogante la determinan las cuatro variables que componen el diamante, estas influyen en la capacidad de las empresas para mantener la ventaja competitiva en los mercados internacionales.

El modelo de la ventaja competitiva nacional está basado en el análisis de las características nacionales que se clasifican en cuatro grupos: las condiciones de los factores, las condiciones de la demanda, las industrias relacionadas y de apoyo y la estructura y rivalidad de las empresas. Los factores deben estar asociados a las decisiones de los gobiernos como determinantes para la competitividad, creando así un entorno propicio para el crecimiento de las empresas (Furman, Porter y Stern, 2002).

Porter (1990) define los factores de competitividad como se muestra en la Tabla 1.

Tabla 1. Factores del diamante de la competitividad

Table 1. Diamond of competitiveness factors

Factor

Condiciones de los factores

Condiciones de la demanda

Las industrias
relacionadas y de apoyo
las empresas

\section{Descripción}

Las naciones crean sus factores más importantes. Porter los clasifica en dos grandes grupos; los factores básicos (tradicionales) y los factores avanzados (tecnológicos) (Porter, 1990).

El estado de la demanda doméstica presiona a las compañías a innovar y mejorar, para Michel Porter el estado actual de las industrias determinan el grado de sofisticación, haciendo que las empresas satisfagan las necesidades emergentes con mercados domésticos más exigentes. (Chacón y Parada, 2005).

Para Porter resulta inconveniente que las empresas o proveedores solo dependan de la industria nacional. Las industrias deben apoyarse creando una cadena de suministro competitiva, no solo para satisfacer la demanda local sino también la extranjera (Porter, 2008).

Las economías con bajos niveles de competitividad se caracterizan por tener poca rivalidad doméstica, la cual presiona intensamente el desarrollo de la industria o sector induciendo a las empresas a buscar la reducción de costos, mejorar la calidad de sus productos y ofrecer mejores servicios a los demandantes (Chacón y Parada, 2005; Furman et al., 2002; Porter, 2008).

Fuente: Elaboración propia basado en (Chacón y Parada, 2005; Furman et al., 2002; M. E. Porter, 2008; M. Porter, 1990). 
Tabla 3. Pilares para la medición de la competitividad

Table 3. Pillars for measuring competitiveness

PILAR

Rendimiento macroeconómico y financiero

Dinámica del mercado Internacional

Innovación y tecnología

Recurso Humano

\section{DESCRIPCIÓN}

Desde el punto de vista del desarrollo de la capacidad productiva de oferta y demanda las variables macroeconómicas representan una relación particular con la competitividad, es decir son variables que por sí mismas ya reflejan el crecimiento económico de las naciones (Anderson Y Torres, 2011; Porter, Sachs y Warner, 2014).

Con la globalización, los mercados internacionales se convierten en sustitutos de los mercados nacionales, sobre todo en los países pequeños. Una vasta apertura comercial es asociada positivamente al crecimiento económico de estas naciones por contener mercados domésticos pequeños. Por tanto, la dinámica de importaciones y exportaciones y las variables que determinen dicha dinámica son sustituto de la demanda interna impulsando el crecimiento (Schwab y WEF, 2012).

A finales de la década de 1980, las ideas de Schumpeter centraron a los economistas en que la innovación y la difusión eran las fuentes de crecimiento económico y, la acumulación de capital físico se convierte en una de las variables endógenas (Fagerberg, Srholec y Knell, 2007).

Los recursos humanos son el conjunto de las características que ayudan a las empresas a obtener ventajas competitivas en los mercados extranjeros. Es necesario contar con mano de obra calificada. (Castro-Gonzáles et al., 2014; Guan, Yam, Mok, y Ma, 2006).

Es la infraestructura básica para la economía y la vida social de cualquier país. Aunque estos indicadores no están relacionados con las capacidades industriales y la producción de conocimiento, están fuertemente asociados a su disponibilidad y difusión. El uso frecuente de las tecnologías de la información y las comunicaciones permiten acortar distancias entre el comprador y el vendedor. (Carvalho et al., 2012; Castro-Gonzáles et al., 2014).

La institucionalidad tiene un fuerte papel en el desarrollo de la competitividad y el crecimiento de las economías, ya que este es el marco regulatorio y político que influye en la toma de decisiones y en la interacción de las empresas, la demanda y el gobierno. No solo el marco regulatorio hace parte del marco institucional sino también las actitudes gubernamentales hacia el trato de las libertades comerciales, la seguridad es un componente indispensable en las inversiones por lo tanto el gasto público en seguridad tiene gran influencia en la inversión (Kao et al., 2008; Porter et al., 2014; Schwab y WEF, 2012), (Aubert, 2004).

Fuente: Elaboración propia.

\section{RESULTADOS Y DISCUSIÓN}

La Organization for Economic Cooperative and Development (OCDE, Commission European, Centre Joint Research y OECD, 2008) proponen una metodología para la construcción de índices compuestos y es apoyada por la metodología usada por (Schuschny y Soto, (2009) para la construcción de índices compuestos de sostenibilidad. La metodología está determinada por los pasos presentados en la Fig. 1, donde se muestra el procedimiento a seguir para la obtención de resultados. 
Tabla 2. Índices de competitividad

Table 2. Competitiveness index

ÍNDICE

$\quad$ INDICE
El Índice de
Competitividad
Global


IMD World
Competitiveness
Yearbook

Es referente para la medición e impulso de las economías. Proporciona causas acerca de la productividad o prosperidad de las naciones, existen diversas opiniones que revelan falencias en el constructo metodológico de este índice, induciendo errores para los tomadores de decisiones. Este índice evalúa el panorama de competitividad de 148 economías, proporcionando información sobre las causas de su productividad y la prosperidad. La serie del informe sigue siendo el estudio más completo de la competitividad nacional en todo el mundo.

Desarrollado año a año por el Instituto Internacional para el Desarrollo Gerencial, (Institute for Management Development, IMD), mide el rendimiento de las naciones con base en cuatro grandes grupos de factores para la competitividad, desempeño económico, eficiencia gubernamental, eficiencia empresarial e infraestructura. El eje principal de este análisis es constituido por dos tipos de variables: en primer lugar 130 variables cuantitativas constituidas por indicadores estadísticos tomados de las fuentes nacionales o multilaterales y en segunda instancia, 116 variables cualitativas obtenidas por encuestas de opinión ejecutiva que es aplicadas por instituciones pares alrededor del mundo.

Recopila información industrial con una gran cantidad de indicadores para 87 países, donde se tienen en cuenta las fuerzas motrices del rendimiento industrial.

Industrial Development Scoreboard Considera cuatro categorías: (a) el esfuerzo tecnológico, (b) el rendimiento competitivo industrial, (c) la importación de alta tecnología y (d) las capacidades y la infraestructura. Con estos componentes no se crea un indicador sintético que los agrupe, por el contrario son considerados individualmente.

En el esfuerzo por mejorar la competitividad de Colombia, en 2005 nace el Sistema Nacional de Competitividad, que luego entra a llamarse, el Sistema Nacional de Competitividad e Innovación (CNCel). Este es un espacio articulado entre lo público y lo privado que pretende medir y monitorear la agenda de competitividad del país. El gobierno colombiano instaura al Consejo Privado de Competitividad (CPC) como el veedor de la agenda y su función es acompañar al gobierno con la implementación de políticas que mejoren la competitividad nacional. La idea principal de este comité nace de la necesidad de conocer los puntos fuertes y débiles del país, debido a las continuas y no mejoradas mediciones del Índice de Competitividad Global durante los últimos años.

Fuente: Elaboración propia basado en: (Archibugi y Coco, 2005; CPC, 2014; IMD, 2013; Schwab y WEF, 2012). 
Tabla 3. Pilares para la medición de la competitividad

Table 3. Pillars for measuring competitiveness

PILAR

Rendimiento macroeconómico y financiero

Dinámica del mercado Internacional

Innovación y tecnología

Recurso Humano

Institucionalidad

\section{DESCRIPCIÓN}

Desde el punto de vista del desarrollo de la capacidad productiva de oferta y demanda las variables macroeconómicas representan una relación particular con la competitividad, es decir son variables que por sí mismas ya reflejan el crecimiento económico de las naciones (Anderson Y Torres, 2011; Porter, Sachs y Warner, 2014).

Con la globalización, los mercados internacionales se convierten en sustitutos de los mercados nacionales, sobre todo en los países pequeños. Una vasta apertura comercial es asociada positivamente al crecimiento económico de estas naciones por contener mercados domésticos pequeños. Por tanto, la dinámica de importaciones y exportaciones y las variables que determinen dicha dinámica son sustituto de la demanda interna impulsando el crecimiento (Schwab y WEF, 2012).

A finales de la década de 1980, las ideas de Schumpeter centraron a los economistas en que la innovación y la difusión eran las fuentes de crecimiento económico y, la acumulación de capital físico se convierte en una de las variables endógenas (Fagerberg, Srholec y Knell, 2007).

Los recursos humanos son el conjunto de las características que ayudan a las empresas a obtener ventajas competitivas en los mercados extranjeros. Es necesario contar con mano de obra calificada. (Castro-Gonzáles et al., 2014; Guan, Yam, Mok, y Ma, 2006).

Es la infraestructura básica para la economía y la vida social de cualquier país. Aunque estos indicadores no están relacionados con las capacidades industriales y la producción de conocimiento, están fuertemente asociados a su disponibilidad y difusión. El uso frecuente de las tecnologías de la información y las comunicaciones permiten acortar distancias entre el comprador y el vendedor. (Carvalho et al., 2012; Castro-Gonzáles et al., 2014).

La institucionalidad tiene un fuerte papel en el desarrollo de la competitividad y el crecimiento de las economías, ya que este es el marco regulatorio y político que influye en la toma de decisiones y en la interacción de las empresas, la demanda y el gobierno. No solo el marco regulatorio hace parte del marco institucional sino también las actitudes gubernamentales hacia el trato de las libertades comerciales, la seguridad es un componente indispensable en las inversiones por lo tanto el gasto público en seguridad tiene gran influencia en la inversión (Kao et al., 2008; Porter et al., 2014; Schwab y WEF, 2012), (Aubert, 2004).

Fuente: Elaboración propia.

\section{RESULTADOS Y DISCUSIÓN}

La Organization for Economic Cooperative and Development (OCDE, Commission European, Centre Joint Research y OECD, 2008) proponen una metodología para la construcción de índices compuestos y es apoyada por la metodología usada por (Schuschny y Soto, (2009) para la construcción de índices compuestos de sostenibilidad. La metodología está determinada por los pasos presentados en la Fig. 1, donde se muestra el procedimiento a seguir para la obtención de resultados. 


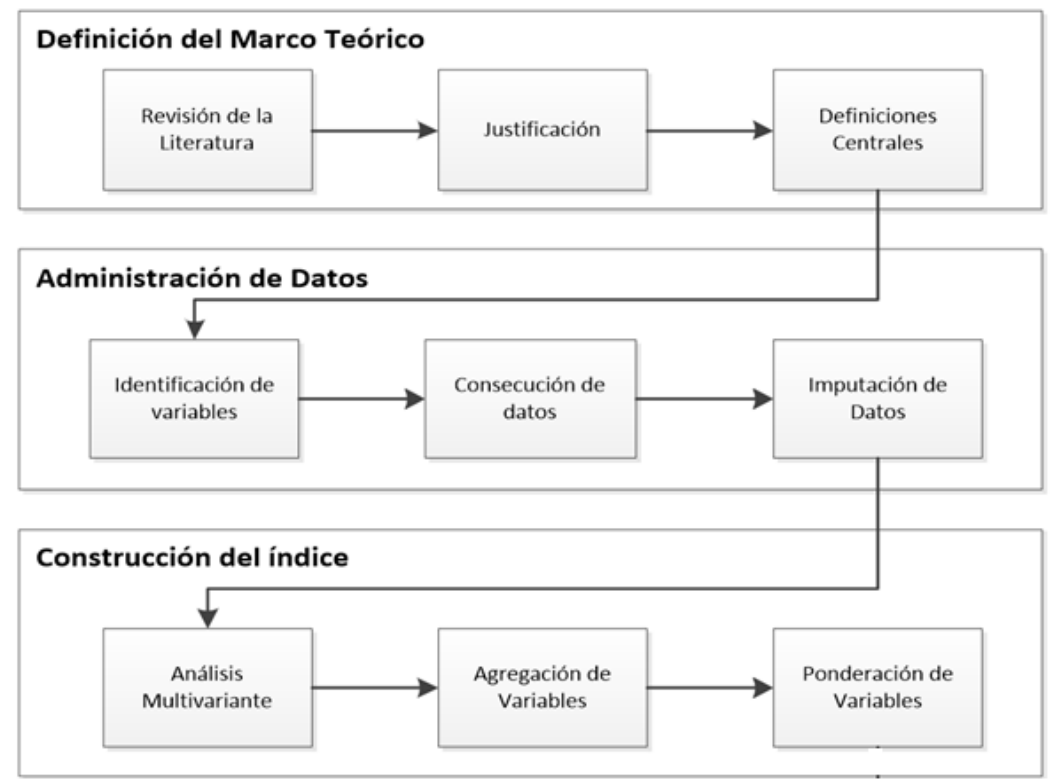

Figura 1. Metodología para la Construcción de Índices compuestos

Figure 1. Methodology for Building Composite index Fuente: Elaboración Propia.

Luego de realizada la revisión literaria pertinente, se continua con la justificación y definición de variables por medio de investigación descriptiva. Una característica importante en las variables seleccionadas es que ninguna es determinada por medio de encuestas o medios que den lugar a la subjetividad, todas son basadas en datos duros debido al matiz objetivo que se desea para este índice. La Tabla 4 muestra las variables introducidas para la construcción del índice y su posterior análisis multivariado.

El análisis factorial es una técnica estadística multivariante que a partir de un conjunto de variables cuantitativas permite determinar un conjunto menor de variables sintéticas o no observables (ficticias), que resumen toda la información que reside en el conjunto original. Estas variables «ficticias» reciben el nombre de factores, y entre sus características se destaca el hecho de encontrarse correlacionadas entre ellas (Landau y Everitt, 2004). Por lo tanto, es una técnica de reducción dimensional con el objetivo de encontrar un número mínimo de dimensiones que expliquen la mayor cantidad de la información.

Esta técnica se encarga de analizar la varianza común entre todas las variables tomando como referencia la matriz de correlaciones, calculando la proporción de varianza compartida determinada por el coeficiente de determinación y simplifica la información existente, esta información es condensada en los llamados factores (De la Fuente, 2011).

Los factores son variables ficticias que recogen la información de la combinación lineal de las variables. Si se comprueba la existencia de $n$ factores, se interpreta que el objeto de estudio puede descomponerse en $n$ factores que reúnen una cantidad determinada de variables.

Contando con los datos completos y asegurando la no existencia de datos atípicos se procedió con el análisis multivariado de los datos. Para encontrar una medida objetiva de la competitividad era necesario que la ponderación y agregación de las variables al modelo no fuera hecha a criterio de expertos, 
sino de acuerdo con el aporte de variabilidad de los datos, por tanto, el método de análisis factorial por medio de extracción por componentes principales fue la herramienta escogida para determinar dichas proporciones, permitiendo agrupar en la menor cantidad de factores las variables y así encontrar un número reducido de componentes que lo componga y expliquen la mayor variabilidad. Al ser un modelo con 36 variables propuestas se requiere de esta herramienta que permita una reducción de la escala y así expresarlo en forma simple.

Tabla 4. Pilares y variables del modelo para la construcción del índice de competitividad

Table 4. Pillars and variables of the model for the construction of the competitiveness index

\begin{tabular}{|c|c|c|}
\hline Pilar & Variable & Fuente \\
\hline \multirow{10}{*}{$\begin{array}{l}\text { Rendimiento } \\
\text { macroeconómico y } \\
\text { financiero del país }\end{array}$} & PIB per cápita en USD & \multirow{16}{*}{$\begin{array}{l}\text { (Benzaquen et al., } \\
\text { 2011; Castro-Gonzáles } \\
\text { et al., 2014; Charles y } \\
\text { Zegarra, 2014; Cho, } \\
\text { Moon, y Kim, 2008; } \\
\text { Durand y Giorno, 1996; } \\
\text { Fernández, Pino Mejías, } \\
\text { Solis Cabrera, y Barea } \\
\text { Barrera, n.d.; Huemer, } \\
\text { Scheubel, y Walch, } \\
\text { 2013; IMD, 2013; Kao } \\
\text { et al., 2008; Sanjaya Lall }\end{array}$} \\
\hline & inflación \% & \\
\hline & Índice GINI (desigualdad) & \\
\hline & Gasto público como porcentaje del PIB & \\
\hline & Balanza de cuenta corriente & \\
\hline & Ahorro nacional & \\
\hline & Formación Bruta de capital & \\
\hline & Capitalización bursátil como porcentaje PIB & \\
\hline & Tipo de Cambio real & \\
\hline & Tasa de interés activa & \\
\hline \multirow{6}{*}{$\begin{array}{l}\text { Mercadeo } \\
\text { Internacional }\end{array}$} & Tasas aduaneras & \\
\hline & Importaciones (sin alta tecnología) & \\
\hline & Exportaciones (sin alta tecnología) & \\
\hline & Inversión Extranjera Directa & \\
\hline & Importaciones alta tecnología & \\
\hline & Exportaciones alta tecnología & \\
\hline \multirow{4}{*}{ Tecnología } & Subsidio a I+D como porcentaje del PIB & \multirow{20}{*}{$\begin{array}{l}\text { y Pietrobelli, 2005; } \\
\text { Sanjaya Lall, 2001; } \\
\text { Mahroum y Al-Saleh, } \\
\text { 2013b; Önsel et al., } \\
\text { 2008; Porter, 1990; } \\
\text { Porter et al., 2014; } \\
\text { Schwab y WEF, 2012; } \\
\text { Unido, 2002; Zanakis y } \\
\text { Becerra-Fernandez, } \\
\text { 2005) }\end{array}$} \\
\hline & Cantidad de investigadores & \\
\hline & Acceso a internet usuarios por cada 100 habitantes & \\
\hline & Patentes & \\
\hline \multirow{7}{*}{ Recurso Humano } & Contratación de género (mujeres respecto a varones) & \\
\hline & Mortalidad (porcentaje por cada 100 personas/año) & \\
\hline & Matriculas en primaria & \\
\hline & Matriculas en secundaria & \\
\hline & Educación terciaria & \\
\hline & Tasa de alfabetización & \\
\hline & Tasa de empleo & \\
\hline \multirow{7}{*}{ Infraestructura } & Ferrocarriles & \\
\hline & Puertos marítimos & \\
\hline & Densidad de transporte aéreo, ton/pasajero & \\
\hline & Demanda de energía, KW per cápita & \\
\hline & Líneas telefónicas por cada 100 personas & \\
\hline & Líneas celular por cada 100 habitantes & \\
\hline & Densidad de carreteras, kilómetros/territorio & \\
\hline \multirow{2}{*}{ Instituciones } & Gasto militar como porcentaje del PIB & \\
\hline & Gasto en Salud & \\
\hline
\end{tabular}

Fuente: Elaboración propia. 
Tabla 6. Matriz de componentes rotadas

Table 6. Component Matrix rotated

\begin{tabular}{|c|c|c|c|c|c|c|}
\hline \multicolumn{7}{|c|}{ Matriz de componentes rotados } \\
\hline \multirow[b]{2}{*}{ Variable } & \multicolumn{6}{|c|}{ Componente } \\
\hline & 1 & 2 & 3 & 4 & 5 & 6 \\
\hline PIB PER CÁPITA & 0,9 & 0,36 & 0,088 & 0,004 & 0,159 & 0,162 \\
\hline INFLACIÓN & $-0,774$ & 0,415 & $-0,246$ & 0,005 & $-0,124$ & 0,386 \\
\hline GASTO PÚBLICO & 0,772 & 0,515 & 0,024 & 0,173 & 0,23 & 0,206 \\
\hline AHORRO NACIONAL & $-0,405$ & $-0,342$ & 0,741 & $-0,274$ & $-0,066$ & $-0,092$ \\
\hline FORMACION BRUTA DE CAPITAL & 0,152 & $-0,225$ & 0,894 & $-0,125$ & 0,144 & $-0,038$ \\
\hline CAPITALIZACIÓN BURSÁTIL & 0,269 & $-0,534$ & $-0,029$ & 0,305 & 0,686 & $-0,142$ \\
\hline TASA DE INTERÉS ACTIVA & $-0,942$ & $-0,109$ & $-0,084$ & $-0,034$ & $-0,252$ & 0,14 \\
\hline IMPORTACIÓN DE BIENES Y SERVICIOS & 0,277 & 0,641 & 0,014 & $-0,624$ & $-0,078$ & $-0,101$ \\
\hline EXPORTACIÓN DE BIENES Y SERVICIOS & $-0,713$ & $-0,249$ & $-0,02$ & $-0,5$ & $-0,292$ & $-0,277$ \\
\hline INVERSIÓN EXTRAJERA DIRECTA & 0,805 & 0,432 & 0,031 & $-0,258$ & 0,242 & 0,18 \\
\hline IMPORTACIONES DE ALTA TECNOLOGÍA & $-0,267$ & $-0,117$ & $-0,033$ & 0,124 & $-0,906$ & $-0,107$ \\
\hline EXPORTACIONES DE ALTA TECNOLOGÍA & $-0,391$ & $-0,492$ & $-0,064$ & 0,586 & $-0,114$ & $-0,269$ \\
\hline ACCESO A INTERNET & 0,934 & 0,287 & $-0,056$ & 0,042 & 0,145 & 0,093 \\
\hline PATENTES & 0,793 & 0,44 & $-0,252$ & $-0,27$ & 0,108 & 0,097 \\
\hline CONTRATACIÓN DE GÉNERO & $-0,199$ & $-0,337$ & $-0,839$ & $-0,213$ & 0,093 & $-0,086$ \\
\hline TASA DE MORTALIDAD & 0,96 & $-0,017$ & $-0,066$ & 0,162 & 0,066 & 0,157 \\
\hline MATRÍCULAS EN FORMACIÓN TERCIARIA & 0,983 & 0,109 & 0,026 & 0,062 & 0,047 & 0,077 \\
\hline TASA DE EMPLEO & 0,953 & 0,169 & 0,051 & $-0,231$ & $-0,063$ & 0,033 \\
\hline TRANSPORTE FÉRREO & 0,923 & 0,088 & 0,178 & 0,112 & 0,223 & 0,069 \\
\hline TRASPORTE MARÍTIMO & 0,929 & 0,286 & 0,078 & $-0,088$ & 0,051 & 0,16 \\
\hline TRANPORTE AÉREO & 0,831 & 0,501 & $-0,142$ & 0,022 & 0,068 & 0,157 \\
\hline LINEAS TELEFÓNICAS & 0,239 & 0,853 & $-0,284$ & $-0,073$ & $-0,225$ & 0,04 \\
\hline LINEAS CELULARES & 0,896 & 0,388 & $-0,07$ & 0,011 & 0,099 & 0,164 \\
\hline GASTO MILITAR & 0,18 & 0,012 & $-0,082$ & 0,948 & $-0,027$ & 0,094 \\
\hline GASTO EN SALUD & 0,96 & $-0,017$ & $-0,066$ & 0,162 & 0,066 & 0,157 \\
\hline INDICE GINI & $-0,908$ & $-0,268$ & 0,147 & 0,087 & $-0,133$ & $-0,048$ \\
\hline CUENTA CORRIENTE & $-0,359$ & $-0,887$ & $-0,074$ & $-0,049$ & $-0,229$ & $-0,007$ \\
\hline INVERSIÓN I+D & 0,72 & 0,185 & 0,167 & 0,261 & 0,325 & 0,406 \\
\hline INVESTIGADORES & 0,757 & $-0,016$ & 0,387 & 0,512 & 0,109 & $-0,038$ \\
\hline TASA DE ALFABETIZACIÓN & 0,578 & 0,336 & $-0,377$ & 0,016 & 0,051 & 0,62 \\
\hline DEMANDA DE ENERGÍA & 0,783 & 0,182 & 0,346 & 0,095 & 0,204 & 0,334 \\
\hline DENSIDAD DE CARRETERAS & $-0,68$ & 0,268 & $-0,184$ & $-0,169$ & $-0,041$ & $-0,6$ \\
\hline
\end{tabular}

Fuente: elaboración propia. 
Tabla 7. Pesos relativos para la ponderación y agregación de variables

Table 7. Weights related to the weighting and aggregation of variables

\begin{tabular}{|c|c|c|c|}
\hline Aporte al factor & Variable & \% saturación & Peso relativo \\
\hline \multirow{21}{*}{$54,10 \%$} & PIB per cápita & 0,9 & 0,05023443 \\
\hline & Inflación & 0,774 & 0,04320161 \\
\hline & Gasto público & 0,772 & 0,04308998 \\
\hline & Tasa de interés activa & 0,942 & 0,0525787 \\
\hline & Exportación de bienes y servicios & 0,713 & 0,03979683 \\
\hline & Inversión extrajera directa & 0,805 & 0,0449319 \\
\hline & Acceso a internet & 0,934 & 0,05213217 \\
\hline & Patentes & 0,793 & 0,04426211 \\
\hline & Tasa de mortalidad & 0,96 & 0,05358339 \\
\hline & Matrículas en formación terciaria & 0,983 & 0,05486716 \\
\hline & Tasa de empleo & 0,953 & 0,05319268 \\
\hline & Transporte férreo & 0,923 & 0,0515182 \\
\hline & Trasporte marítimo & 0,929 & 0,05185309 \\
\hline & Transporte aéreo & 0,831 & 0,04638312 \\
\hline & Líneas celulares & 0,896 & 0,05001116 \\
\hline & Gasto en salud & 0,96 & 0,05358339 \\
\hline & Índice GINI & 0,908 & 0,05068096 \\
\hline & Inversión I+D & 0,72 & 0,04018754 \\
\hline & Investigadores & 0,757 & 0,04225273 \\
\hline & Demanda de energía & 0,783 & 0,04370395 \\
\hline & Densidad de carreteras & 0,68 & 0,0379549 \\
\hline \multirow{3}{*}{$15.33 \%$} & Importación de bienes y servicios & 0,641 & 0,26921462 \\
\hline & Líneas telefónicas & 0,853 & 0,35825283 \\
\hline & Cuenta corriente & 0,887 & 0,37253255 \\
\hline \multirow{3}{*}{$9,33 \%$} & Ahorro nacional & 0,741 & 0,29951496 \\
\hline & Formación bruta de capital & 0,894 & 0,36135812 \\
\hline & Contratación de género & 0,839 & 0,33912692 \\
\hline \multirow{2}{*}{$9,16 \%$} & Exportaciones de alta tecnología & 0,586 & 0,38200782 \\
\hline & Gasto militar & 0,948 & 0,61799218 \\
\hline \multirow{2}{*}{$6,66 \%$} & Capitalización bursátil & 0,686 & 0,43090452 \\
\hline & Importaciones de alta tecnología & 0,906 & 0,56909548 \\
\hline $5,44 \%$ & Tasa de alfabetización & 0,62 & 1,0 \\
\hline
\end{tabular}

Fuente: Elaboración propia. 
Tabla 8. Pilares de la competitividad para Colombia 20112012

Table 8. Pillars of competitiveness for Colombia in 2011 and 2012

\begin{tabular}{lcc}
\multicolumn{1}{c}{ PILAR } & 2011 & 2012 \\
\hline Rendimiento Macro y Financiero & 14,58 & 15,60 \\
\hline Mercado Internacional & 6,53 & 5,22 \\
\hline Tecnología e Innovación & 8,46 & 9,12 \\
\hline Recurso Humano & 8,59 & 9,81 \\
\hline Infraestructura & 12,53 & 13,24 \\
\hline Institucionalidad & 3,03 & 2,70
\end{tabular}

Fuente: Elaboración Propia

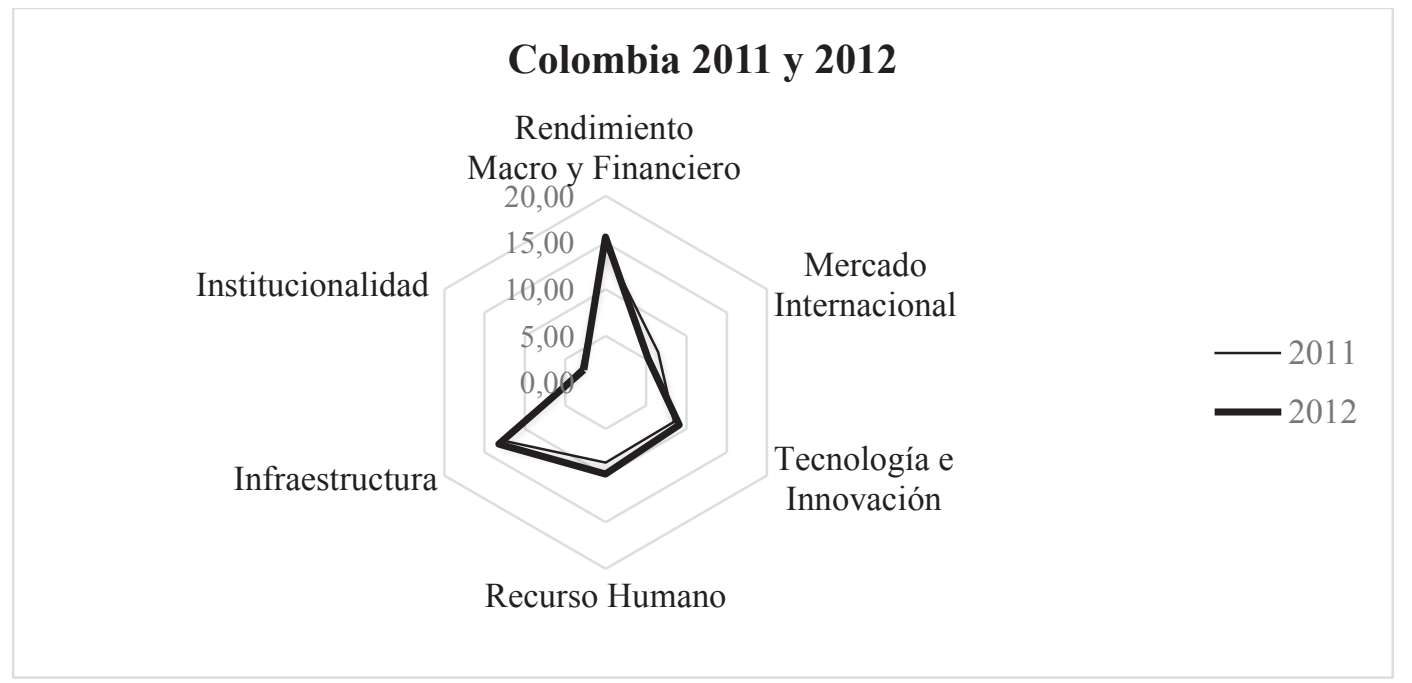

Figura 2. Pilares de la competitividad para Colombia entre 2011 y 2012

Figure 2. Pillars of competitiveness for Colombia between 2011 and 2012

Fuente: Elaboración Propia.

En comparación con los resultados expuestos por los informes económicos de la OCDE, el índice creado en esta investigación se aproxima a la realidad mostrando fortalezas y debilidades en los mismos pilares que se tratan en el informe.

\section{CONCLUSIONES}

La competitividad nacional está dada por un entorno tanto nacional como internacional que interactúan de manera recíproca. Las empresas y los territorios deben trabajar conjuntamente para el desarrollo de ventajas competitivas y posicionar a Colombia como un país apto para la inversión, por tanto, es obligación del territorio el continuo desarrollo de políticas que mejoren los pilares que determinan la competitividad, y además mejorar los esfuerzos hechos de manera agregada.

Por su parte, la competitividad debe ser medida de forma objetiva, ya que de los índices resultantes se derivan decisiones a nivel nacional con el objetivo de alcanzar el desarrollo económico superior. En el caso de esta investigación, el uso de datos duros, provenientes de bases de datos verificables, dota a la misma de carácter objetivo al eludir 
las encuestas que por la falta de un método estandarizado de construcción no permiten medir de forma imparcial las características cualitativas de las economías, específicamente en las variables de innovación.

El índice construido muestra la proximidad a la realidad debido a que los resultados arrojados muestran las fortalezas y debilidades asociadas a la competitividad de Colombia; además, da cuenta de la falencia que tiene el país en aspectos como la infraestructura y en actividades de ciencia y tecnología.

De acuerdo con esta investigación, Colombia tiene problemas que impiden la mejora en la medición de la competitividad. El primero de ellos es la institucionalidad, aunque es uno de los pilares más complejos de medir por tratarse de variables cualitativas, esta investigación lo enfoca en el bienestar y la seguridad de la población. Con respecto la seguridad, se evidencia que el gobierno colombiano hace esfuerzos en términos del gasto militar para proveer seguridad en los habitantes; sin embargo, en la variable salud, sus esfuerzos no son los suficientes, ya que en línea con las recomendaciones hechas por la OCDE ( 2013), la salud es tema que debe tratarse y se hacen avances en materia de ello en los últimos años; los temas de infraestructura que apoyen el bienestar de los ciudadanos como la cobertura universal en servicios públicos.

En términos de infraestructura, se evidencia que el país hace esfuerzos en su mejora; sin embargo comparado con las otras economías en las que se centra esta investigación, la infraestructura sigue siendo escasa; de esta depende el buen desempeño logístico y por ende un desarrollo competitivo, bajando los costos por distribución y almacenamiento del flujo de mercancías. Por ello el CPC (2014) recomienda que los proyectos de construcción se centren en el desarrollo de sistemas de transporte multimodal generados para los principales corredores logísticos del país, además la implementación de mejoras y modernización de los puertos (marítimos y aéreos) para la solución de problemas de capacidad.

\section{REFERENCIAS}

Archibugi, D. y Coco, A. (2005). Measuring technological capabilities at the country level: A survey and a menu for choice. Research Policy, 34(2), 175-194. http://doi.org/10.1016/j.respol.2004.12.002.

Benzaquen, J., del Carpio, L. A., Zegarra, L. A. y Valdivia, C. A. (2011). A competitiveness index for the regions of a country. Cepal Review, (102), 67-84.

Cartwright, W. R. (1993). Multiple Linked "Diamonds" and the International Competitiveness of Export-Dependent Industries: The New Zealand Experience.

Carvalho, L. C. De, Di Serio, L. C. y Vasconcellos, M. A. De. (2012). Competitividade das nações: análise da métrica utilizada pelo World Economic Forum. Revista de Administração de Empresas, 52(4), 421-434. http://doi.org/10.1590/S003475902012000400005 .

Castro-González, S., Peña-Vinces, J., Ruiz-Torres, A. J. y Sosa, J. C. (2014). Estudio intrapaíses de la competitividad global desde el enfoque del doble diamante para Puerto Rico, Costa Rica y Singapur. Investigaciones Europeas de Direccion Y Economia de La Empresa, 20(3), 122-130. http://doi.org/10.1016/j.iedee.2013.09.001

Chacón, W. y Parada, A. M. (2005). Elementos teóricos y metodológicos para el análisis de la competitividad en empresas y sectores. ABRA, 34, 31-51.

Chang Moon, H., Rugman, A. M. y Verbeke, A. (1998). A generalized double diamond approach to the global competitiveness of Korea and Singapore. International Business Review, 7(2), 135-150. http://doi.org/10.1016/S09695931(98)00002-X. 
CPC. (2014). Informe Nacional de Competitividad. Bogotá, Colombia.

De la Fuente, S. (2011). Análisis Factorial. Madrid: Universidad Autónoma de Madrid.

Delgado, M., Ketels, C., Porter, M. E. y Stern, S. (2012). The Determinants of National Competitiveness.

Dunning, J. H. (2005). Is global capitalism morally defensible? Contributions to Political Economy, 24(1), 135-151. http://doi.org/10.1093/cpe/bzi007.

Furman, J., Porter, M. y Stern, S. (2002). The determinants of national innovative capacity. Research Policy, 31(2002), 899-933.

Grant, R. (1991). The resource-based theory of competitive advantage: Implications for strategy formu- lation. Knowledge and strategy. Woburn: Butterworth-Heinemann.

IMD. (2013, August). IMD business school, Switzerland.

Kao, C., Wu, W. Y., Hsieh, W. J., Wang, T. Y., Lin, C. Y Chen, L. H. (2008). Measuring the national competitiveness of Southeast Asian countries. European Journal of Operational Research, 187(2), 613-628. http://doi.org/10.1016/j.ejor.2007.03.029.

Krugman, P. y Obstfeld, M. (2000). International Economics, Theory and Practice. Adddison Wesley Longman.

Lall, S. (2001). Competitiveness indices and developing countries: An economic evaluation of the global competitiveness report. World Development, 29(9), 1501$1525 . \quad$ http://doi.org/10.1016/S0305750X(01)00051-1.

Lall, S. y House, Q. (2001). Comparing National Competitiveness Performance: An Economic Analysis of World Economic Forum's Competitive Index, (61).

Landau, S. y Everitt, B. (2004). A handbook of statistical analyses using SPSS.

Lira, I. S. (2005). Desarrollo económico local y competitividad territorial en América Latina. Revista de La CEPAL. Retrieved from http://repositorio.cepal.org/handle/11362/1 1001.

OCDE. (2013). Estudios económicos de la OCDE.

OCDE, Commission European, Centre Joint Research y OECD. (2008). Handbook on constructing composite indicators: methodology and user guide. Disponible en http://cna.bnv.edu.cn/docs/2014060510172 3366354.pdf.

Önsel, Ş., Ülengin, F., Ulusoy, G., Aktaş, E., Kabak, Ö. y Topcu, Y. I. (2008). A new perspective on the competitiveness of nations. SocioEconomic Planning Sciences, 42(4), 221246. http://doi.org/10.1016/j.seps.2007.11.00 1.

Porter, M. (1990). La competitividad de las naciones. Buenos Aires Argentina.

Porter, M. E. (2008). The five competitive forces that shape strategy. Harvard Business Review, 86(1), 78-93, 137. http://doi.org/Article.

Ramos, R. (2001). Modelos de evaluación de la competitividad internacional. Una aplicación empírica al caso de las Islas Canarias. Universidad de las Palmas de Gran Granada.

Schuschny, A. y Soto, H. (2009). Guía metodológica Diseño de indicadores compuestos de desarrollo sostenible Andrés Schuschny. Cepal, 109.

Schwab, K. y WEF. (2012). The global competitiveness report 2012-2013. Retrieved from

http://www.weforum.org/pdf/Global_Compe titiveness_Reports/Reports/factsheet_gcr03. pdf.

Weymouth, S. y Feinberg, R. (2011). National competitiveness in comparative perspective: evidence from Latin America. Latin American Politics and Society.

Xia, R., Liang, T., Zhang, Y. y Wu, S. (2012). Is global competitive index a good standard to measure economic growth? A suggestion for improvement. International Journal of Services and Standards, 8(1), 45. http://doi.org/10.1504/IJSS.2012.048438. 